\title{
Theoretical Clarification of Facet like Fracture in Hydrogen Embrittlement"
}

\author{
Shinya TAKETOMI ${ }^{* *}$ and A. Toshimitsu YOKOBORI** \\ **Department of Nanomechanics, Tohoku University, \\ 6-6-01, Aza-Aoba, Aramaki, Aobaku, Sendai, 980-8579, Japan \\ E-mail: yokobori@md.mech.tohoku.ac.jp
}

\begin{abstract}
Based on a two-dimensional physical model of a mode I crack with a slip band, the analysis of mechanical interaction between hydrogen and dynamic dislocations with non-steady emission from a stressed source around a crack tip is conducted. To analyze the mechanical interaction between a crack and dynamic dislocations on a neighboring slip band, a crack is replaced by static discrete dislocations mechanically equivalent with the crack and a physical model of analyzing the mechanical interaction between static, dynamic dislocations and hydrogen is constructed. The occurrence conditions of the mechanisms of corrosive anodic chemical reaction, inter-granular cracking due to hydrogen concentration and facet like fracture due to the interaction between hydrogen and dynamic dislocations were clarified. These results were found to be in good agreement with previous results based on a convenient one-dimensional model. Furthermore, the competitive interaction between hydrogen and dynamic dislocations which causes the facet like fracture is found to occur under the limited velocity range of hydrogen and dynamic dislocations which is closely related to the corresponding range of yield stress.
\end{abstract}

Key words: Hydrogen Embrittlement, Dislocation, Fracture Criterion, Numerical Analysis

\section{Introduction}

Hydrogen embrittlement mechanisms have been theoretically investigated based on hydrogen diffusion theory for the welded metals ${ }^{(1),(2)}$ and materials with cracks $^{(3)-(7)}$. According to these studies, it was pointed out that high strength steels, especially the materials with the yield stress over $1000 \mathrm{MPa}$, are severely suffered from the hydrogen embrittlement due to the local hydrogen accumulation at the high hydrostatic stress region $\left(\sigma_{p}=\left(\sigma_{x}+\sigma_{y}+\sigma_{z}\right) / 3\right)^{(6),(7)}$. The occurrence of this hydrogen embrittlement mechanism attributes to the high hydrogen diffusivity for the materials with high yield stress, since the hydrogen accumulation is dominated by the local gradient of hydrostatic stress. In fact, it was indicated such hydrogen embrittlement fracture occurs for the steels with high strength, typically over $1000 \mathrm{MPa}$ yield stress, under corrosive conditions ${ }^{(7),(8)}$. Hydrogen atoms are induced by the anodic dissolution under corrosive conditions and the hydrogen atom penetrates into the metals, after that hydrogen accumulates at the elastic-plastic boundary around the crack tip due to the steep gradient of hydrostatic stress for high strength steels. In contrast, the ductile materials with lower yield stress (materials with the yield stress below $400 \mathrm{MPa}$ ) have the gradual gradient of hydrostatic stress around the crack tip, thus the typical hydrogen accumulation does not occurs and the fracture mechanism is dominated by the anodic dissolution ${ }^{(7),(8)}$.

\footnotetext{
${ }^{*}$ Received 20 Aug., 2009 (No. T2-08-0020) Japanese Original : Trans. Jpn. Soc. Mech. Eng., Vol. 74, No. 745, A (2008), pp.1271-1277 (Received 15 Jan., 2008) [DOl: 10.1299/jmmp.4.12]
}

Copyright $\odot 2010$ by JSME 
On the other hand, it was reported that the facet like cleavage fracture sometimes occurs under hydrogen environmental conditions including corrosive conditions ${ }^{(9)}$. This fracture was typically observed for the materials with intermediate yield stress ${ }^{(9)}$, and the authors explained the fracture mechanism based on the interaction model between dislocations and hydrogen ${ }^{(9)}$. However, this fracture model was practically the same as that for ductile Hall-Petch law, it attributes to the piled up dislocations against the grain boundary. Concerning the mechanism of transgranular cleavage fracture, dislocation free zone (DFZ) was noticed as a trigger point based on the analysis of dislocation group dynamics around a crack tip, where the inverse pile-up of dislocations results in cleavage fracture ${ }^{(10),(11)}$. It was in good agreement with the experimentally obtained relationship between trigger point and fracture toughness ${ }^{(10),(11)}$. These facts imply that facet like cleavage fracture under hydrogen environmental conditions is dominated by the inverse pile-up of dislocations at the trigger point ahead of the crack tip. Since the velocities of dislocation motion and hydrogen diffusion is supposed to be the competitive relationship for the materials with intermediate yield stress $(400 \sim 1000 \mathrm{MPa})$, it is necessary to clarify the occurrence mechanism of the typical pile-up of dislocations due to the interaction between dislocations and hydrogen. We reported the typical inverse pile-up of dislocations which results in the facet like cleavage fracture occur due to the mechanical interaction between dislocations and hydrogen based on the approximated one-dimensional physical model using the mathematically similar fundamental equation ${ }^{(12)-(14)}$.

In this paper, two-dimensional analysis as an actual physical model of the mechanical interaction between moving dislocations and hydrogen is conducted, and the results obtained in this analysis are compared with those obtained in the previous approximated physical model. The two-dimensional physical model is not feasible to perform the analysis due to its complexity of the model. Therefore, if the results obtained in this analysis model are in good agreement with the approximated physical model such as one-dimensional model, the analysis could perform much easier. Furthermore, the dominant hydrogen embrittlement mechanism for various conditions can be predicted easily, and is very valuable for the engineering viewpoint.

\section{Analysis model and fundamental equations}

\subsection{Physical model}

In this study, analyses are performed using the realistic physical model of a crack and dislocations emitted from a crack tip as shown in Fig.1. In this model, slip-lines are originated in the direction of $\pi / 4$ from a crack tip corresponding to the direction of maximum shear stress. Dislocation dynamics analysis near a crack tip usually introduces the effect of mirror force of emitted dislocation in order to take the boundary condition of crack free surface into consideration ${ }^{(10)}$. Meanwhile, in this paper, a mode I type crack was replaced by infinitesimal continuum distribution of edge dislocations ${ }^{(15)}$. Therefore, the continuum distribution of these dislocations is replaced by the distribution of discrete dislocations in order to keep the mechanical equivalence with continuum distribution of dislocations. The assurance of mechanical equivalence between them is confirmed by investigating the stress singularity described later.

Since this physical model is symmetrical to the horizontal axis, the analyses are conducted based on the approximated physical model as shown in Fig.2. In this model, a dislocation source $\mathrm{S}$ is located at some finite distance $x_{\mathrm{s}}$ on the slip line from a crack tip. The value of $x_{\mathrm{s}}$ is same as in our previous paper ${ }^{(10)} ; x_{\mathrm{s}}=10^{-6} \mathrm{~mm}$. The dislocations on the slip line are emitted from the dislocation source when the effective stress exerted on the dislocation source becomes dislocation source activation stress under local stress field of a 


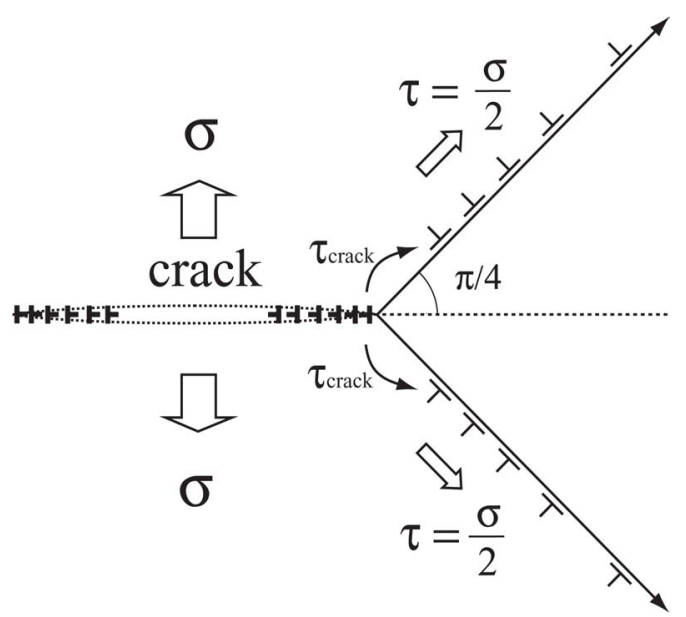

Fig.1 Physical model of a crack and discrete dislocations emitted from a crack tip

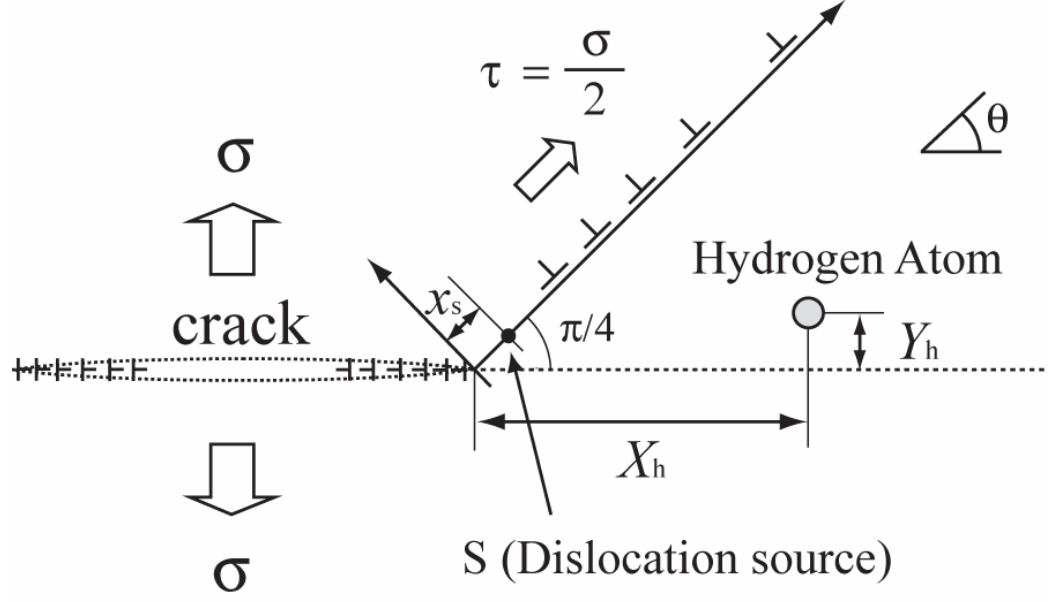

Fig.2 Two-dimensional physical model of mechanical interaction between discrete dislocations and hydrogen around a crack tip

crack, and dislocations move away from a crack on the slip plane. Each dislocation on the slip line is affected by the other dislocations on the same slip plane, a hydrogen atom positioned at $\left(X_{\mathrm{h}}, Y_{\mathrm{h}}\right)$ and the discrete dislocations distributed to simulate the stress singularity field of a crack as shown in Fig.2.

\subsection{Stress field around a crack tip replaced by discrete dislocations}

In the two-dimensional physical model as shown in Fig.1, stress field of a mode I crack is replaced by the discrete dislocations. The stress field of an edge dislocation is given by Peach-Koehler equations as follows.

$$
\begin{aligned}
& \sigma_{x}=-A \frac{y\left(3 x^{2}+y^{2}\right)}{\left(x^{2}+y^{2}\right)^{2}} \\
& \sigma_{y}=A \frac{y\left(x^{2}-y^{2}\right)}{\left(x^{2}+y^{2}\right)^{2}} \\
& \tau_{x y}=A \frac{x\left(x^{2}-y^{2}\right)}{\left(x^{2}+y^{2}\right)^{2}}
\end{aligned}
$$

where, $A=\mu b / 2 \pi(1-v), \quad \mu$ : shear modulus, $b$ : Burger's vector and $v$ : Poisson's ratio. Stress field of a mode I crack is equivalently replaced by continuous distribution of infinitesimal dislocations ${ }^{(16)}$ as 
shown in Fig.3. And the dislocation density becomes infinity at the both crack tips (infinity of dislocation density function). We already analyzed ${ }^{(17)}$ the mechanical interaction between dislocations and a hydrogen atom which results in a typical inverse pile-up of dislocations, and reported that the stress singularity of piled up dislocation is same as that of a crack. Based on this concept, mode I crack is equivalently replaced by discrete dislocations in this analysis. Specifically, discrete dislocations are positioned at the locations of piled up dislocations with dislocation density singularity as $r^{-1 / 2}$. The dislocation density becomes 243 times larger at the crack tip compared with the crack center. This dislocation distribution gives the same singularity of local stress field of a crack. The number of discrete dislocations in this model is 98 .

Shear stress exerted on $\left(x_{i}, y_{i}\right)$ on a slip line by the equivalent discrete dislocations in the right half part of a mode I crack is given by the equation(2) by using the Peach-Koehler force (Fig.4(a)).

$$
\tau_{\text {right,i }}=\sum_{j=1}^{\mathrm{N}}\left[\frac{2 A\left(x_{i}-x_{j}\right) y_{i}^{2}}{\left\{\left(x_{i}-x_{j}\right)^{2}+y_{i}^{2}\right\}^{2}} \sin 2 \theta-A \frac{y_{i}\left\{y_{i}^{2}-\left(x_{i}-x_{j}\right)^{2}\right\}}{\left\{\left(x_{i}-x_{j}\right)^{2}+y_{i}^{2}\right\}^{2}} \cos 2 \theta\right]
$$

Similarly, the shear stress exerted on $\left(x_{i}, y_{i}\right)$ by the equivalent discrete dislocations in the left part of a mode I crack is given by the equation(3).

$$
\tau_{\text {left }, \mathrm{i}}=\sum_{j=\mathrm{N}+1}^{2 \mathrm{~N}}\left[\frac{2 A\left(x_{i}-x_{j}\right) y_{i}^{2}}{\left\{\left(x_{i}-x_{j}\right)^{2}+y_{i}^{2}\right\}^{2}} \sin 2 \theta+A \frac{y_{i}\left\{y_{i}{ }^{2}-\left(x_{i}-x_{j}\right)^{2}\right\}}{\left\{\left(x_{i}-x_{j}\right)^{2}+y_{i}^{2}\right\}^{2}} \cos 2 \theta\right]
$$

According to the equaions (2) and (3), shear stress exerted on $\left(x_{i}, y_{i}\right)$ in a slip line is given by the equation(4).

$$
\tau_{\text {crack,i }}=\tau_{\text {right,i }}+\tau_{\text {left,i }}
$$

Shear stress singularity obtained by the distribution of discrete dislocations to simulate the mode I crack is compared with that of a macroscopic mode I crack (stress distribution around a crack tip is given by equation(5) based on linear fracture mechanics) as shown in Fig.5.

$$
\begin{aligned}
\tau & =\frac{K}{\sqrt{2 \pi r}} f(\theta) \\
& =A \times \frac{1}{r^{0.5}}
\end{aligned}
$$

These results show that the stress singularity based on equivalent discrete dislocations is found to be in good agreement with that obtained by a macroscopic mode I crack based on linear fracture mechanics.

\subsection{Fundamental equations}

The analysis based on the two-dimensional physical model (Fig.2) is conducted. The effective stress exerted on the $i$ th dislocation is written by equation(6).

$$
\begin{aligned}
\tau_{\text {eff }, i}= & \frac{\dot{\sigma}}{2} \mathrm{t}+\tau_{\text {crack }}+A \sum_{\substack{i=1 \\
i \neq j}}^{\mathrm{n}} \frac{1}{s_{i}-s_{j}} \\
& -\frac{2 A^{*} \frac{X_{\mathrm{h}}-Y_{\mathrm{h}}}{\sqrt{2}}\left(s_{i}-\frac{X_{\mathrm{h}}+Y_{\mathrm{h}}}{\sqrt{2}}\right)}{\left\{\left(s_{i}-\frac{X_{\mathrm{h}}+Y_{\mathrm{h}}}{\sqrt{2}}\right)^{2}+\left(\frac{X_{\mathrm{h}}-Y_{\mathrm{h}}}{\sqrt{2}}\right)^{2}\right\}^{2}},
\end{aligned}
$$

where, $\dot{\sigma}$ is the increasing rate of applied tensile stress, $s_{i}$ is the distance from the crack tip to the $i$ th dislocation along the slip line; $s_{i}=\left(X_{i}^{2}+Y_{i}^{2}\right)^{0.5}$, and $A^{*}$ is the constant which represents the magnitude of the mechanical interaction force between hydrogen and 
dislocation. Hence, the first term represents the applied shear stress, the second term is the shear stress exerted on a slip line by a crack replaced by equivalent discrete dislocations, the third term is the mechanical interaction stress between dislocations in the dislocation arrays, and the fourth term is the mechanical interaction stress between dislocations and a hydrogen atom.

The relationship between effective stress $\tau_{\text {eff, } i}$ and dislocation velocity $V_{i}$ is written for each individual dislocation in a coplanar array of dislocation groups by equation(7).

$$
\begin{gathered}
V_{i}=M \tau_{\mathrm{eff}, i}{ }^{m}, \\
M=V_{0}\left(1 / \tau_{0}^{*}\right)^{m},
\end{gathered}
$$

where $\tau_{0}{ }^{*}$ is the specific shear stress required to move an isolated dislocation with a velocity of $0.01 \mathrm{~m} / \mathrm{s}$; and $V_{0}$ is $0.01 \mathrm{~m} / \mathrm{s}$. Analytical methods are the same as our previous analyses $^{(10)-(13)}$.

\section{Analysis result}

\subsection{Analysis based on two-dimensional physical model}

The geometrical relationship between dislocations and a hydrogen atom around a crack tip is shown in Fig.6. Distribution behaviors of dislocation density on the slip line obtained by two-dimensional approximated physical model are shown in Fig.7(a) to (c). The dislocation density $f_{d}$ is defined as following equation(9).

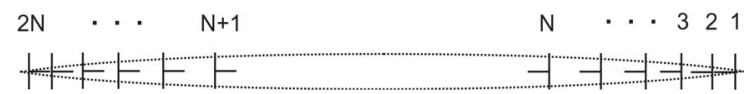

Fig.3 Continuum distribution of infinitesimal dislocations equivalent with a mode I type crack

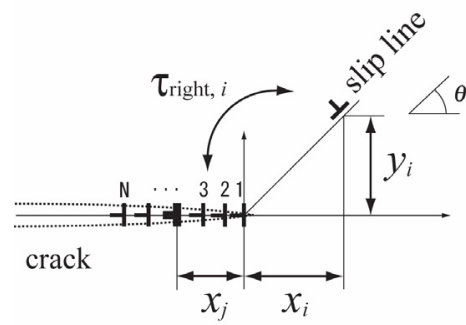

(a) Half right hand part of a mode I crack

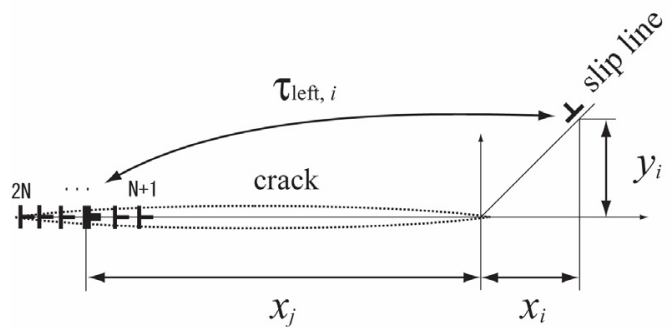

(b) Half left hand part of a mode I crack

Fig.4 Shear stress exerted at the site of $\left(x_{i}, y_{i}\right)$ in a slip line due to discrete dislocations of a mode I crack 


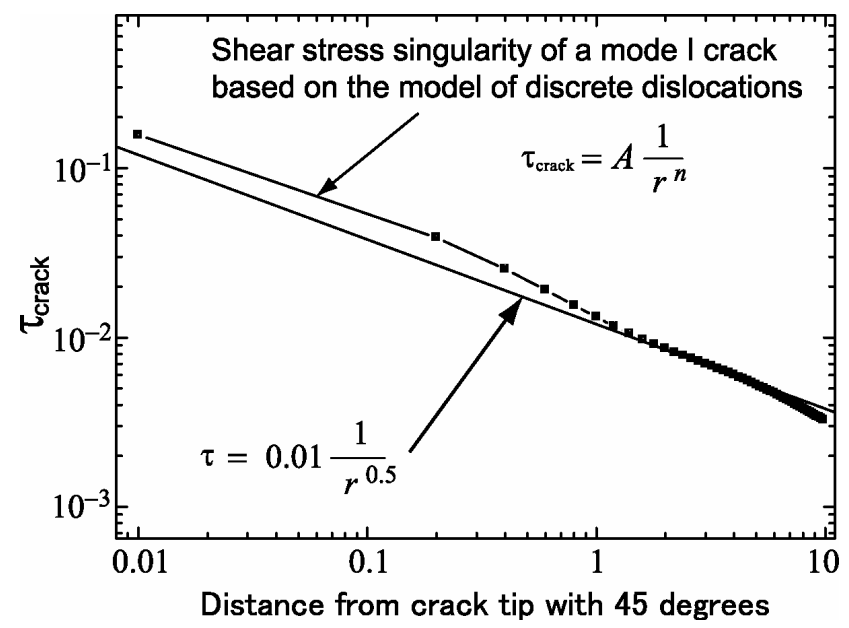

Fig.5 Comparison of the stress singularity in the direction of the slip line from a crack tip obtained by equivalent discrete dislocations of a mode I crack with that of a macroscopic mode I crack based on linear fracture mechanics

$$
f_{d}\left(x_{i+1 / 2}\right)=\frac{1}{x_{i}-x_{i+1}},
$$

where, $x_{i}$ is the distance of the $i$ th dislocation from the crack tip. For ductile materials with lower yield stress, since the diffusion rate of hydrogen is lower which attribute to the gradual gradient of hydrostatic stress field around a crack, hydrogen atom does not diffuses and stably exists around a crack tip. In contrast, dislocation mobility is high for ductile materials, therefore the dislocation moves ahead of the hydrogen as shown in Fig.6(a). Note that the mechanical interaction between dislocations and hydrogen is very small and dislocations distribute as similar as that in the absence of the mechanical interaction between dislocations and hydrogen as shown in Fig.7(a). For brittle materials with higher yield stress, since the gradient of hydrostatic stress around a crack tip is steep, the diffusion rate of hydrogen becomes high. Therefore, hydrogen atoms diffuse to the maximum hydrostatic stress region, and the hydrogen atom exists around the crack plane direction. In contrast, dislocation mobility is low for brittle materials, therefore the dislocation moves very slowly as shown in Fig.6(c). The mechanical interaction between dislocations and hydrogen is also very small for brittle materials, and the dislocations distribute as similar as that in the absence of the mechanical interaction between dislocations and hydrogen as shown in Fig.7(c). On the other hand, for ductile materials with higher yield stress (intermediate yield stress), the driving force of hydrogen diffusion around a crack and the mobility of dislocation are at the state of competitive relationship each other. Thus, some of hydrogen atoms may diffuse toward the direction of slip line in this case. Therefore the hydrogen atoms are positioned near the slip line as shown in Fig.6(b). For this case, typical inverse pile-up of dislocations at the site of hydrogen atom was found to be caused as shown in Fig.7(b). This phenomenon attributes to the mechanical interaction between dislocations and hydrogen due to the formation of Cottrell atmosphere as same as our previous study $^{(12-14)}$. 


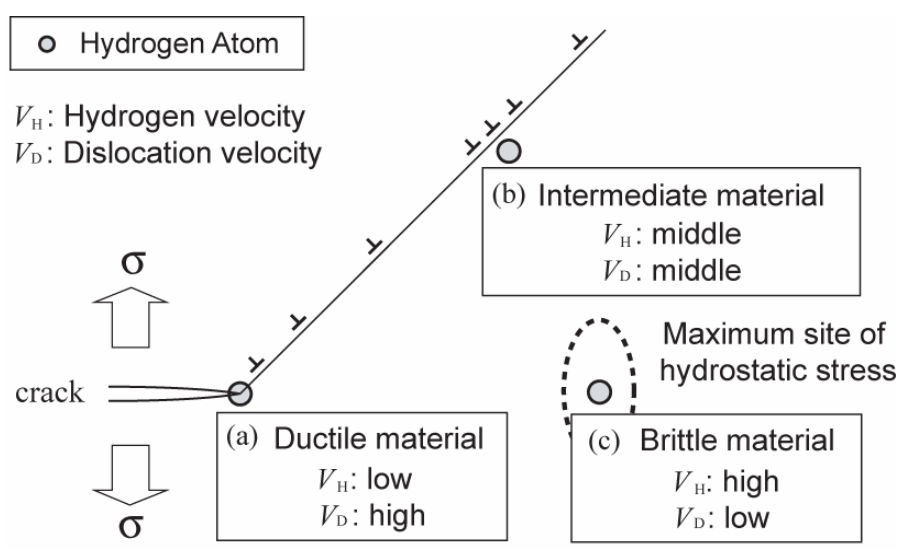

Fig.6 Geometrical relation of crack, dislocations and hydrogen for yield stress of the materials

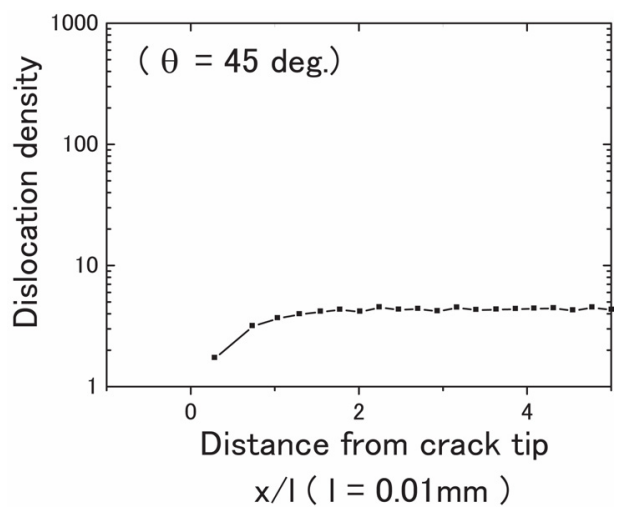

(a) Ductile material

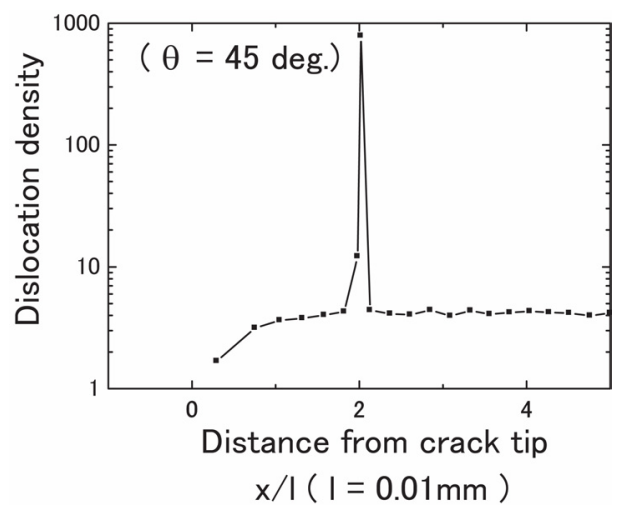

(b) Intermediate material

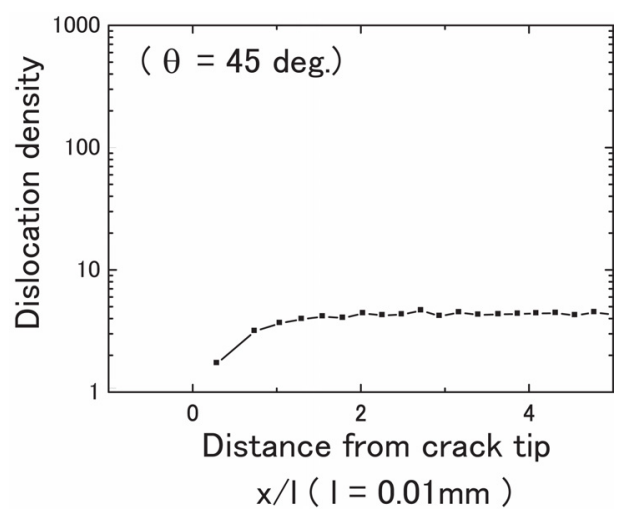

(c) Brittle material

Fig.7 Dislocation density distribution around a crack tip on a slip line. 
The comparisons of the maximum value of the dislocation density between the two-dimensional analysis model and the one-dimensional analysis in our previous study[12-14] are shown in Fig.8. These results reveals that the maximum dislocation density obtained by two-dimensional physical model is quantitatively in good agreement with the results obtained in one-dimensional physical model analyses ${ }^{(12)-(14)}$. According to this fact, the simplified one-dimensional physical model analyses ${ }^{(12)-(14)}$ lead the quantitatively the same results compared with the two-dimensional analysis in this study. It attributes to the similarity of the fundamental equations of these physical models from mathematical point of view. In the Fig.8, results of analyses based on one-dimensional analysis model with moving hydrogen (specific diffusion velocity is given) ${ }^{(13)}$ are also shown. It is clearly shown that the typical inverse pile-up of dislocations occur when the velocities of leading dislocation and hydrogen diffusion are at the state of competitive relation, and the maximum value of the dislocation density is quantitatively the same as that obtained by two-dimensional physical model (the maximum value of the hatching region in Fig.8). Moreover, the maximum dislocation density rapidly decreases to the value without the interaction with hydrogen when the relationship between dislocations and hydrogen are other than competitive condition (the minimum value of the hatching region in Fig.8). These results clearly show that typical inverse pile-up of dislocations occurs due to the mechanical interaction between hydrogen and dislocations when the diffusion rate of hydrogen and the velocity of dislocations are at the state of competitive relationship, and which results in facet like fracture. Moreover the validity of the one-dimensional approximated physical model is assured to be valid not only qualitative but also quantitative point of view.

\subsection{Analysis based on one-dimensional physical model}

The validity of one-dimensional analysis result was shown in the previous chapter, thus, the detailed analyses are performed in order to clarify the occurrence condition of the mechanical interaction between hydrogen and dislocations. The correlation between the maximum dislocation density and the distance of hydrogen atom from slip line, RP, is shown in Fig.9.

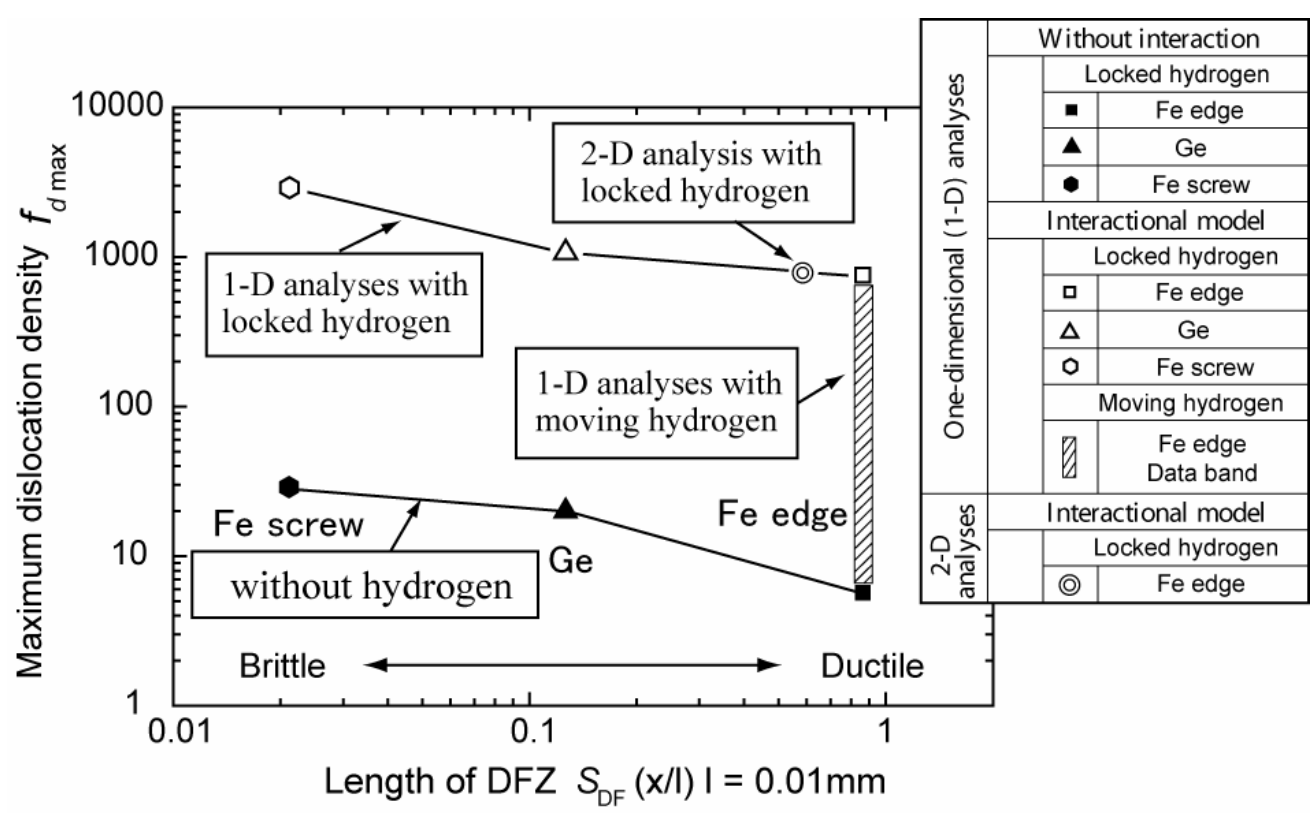

Fig. 8 The maximum dislocation density of the inverse pile-up obtained by one and two-dimensional analysis 
The mechanical interaction does not occur when a hydrogen atom exists far away from the slip line, however, the interaction occurs when the value of RP becomes less than $1.5 \times$ $10^{-2}\left(1.5 \times 10^{-1} \mu \mathrm{m}\right)$ and results in typical inverse pile-up of dislocations as is shown in dark region of Fig.9. The vertical line in Fig.9 corresponds to the Cottrell atmospheric distance. This result suggests the existence of the effective distance of hydrogen position from the slip line to have mechanical multi-interaction between dislocations and hydrogen cluster. The typical inverse pile-up of dislocations only occurs when the dislocations and hydrogen are in this effective region.

The analysis of the mechanical interaction between moving dislocations and moving hydrogen around a crack tip under constant stress rate condition is conducted to estimate the correlation between the maximum dislocation density and hydrogen diffusion velocity. In this analysis the hydrogen diffusion velocity is given $\alpha$ times as large as the leading dislocation velocity, and the hydrogen is assumed to diffuse along the slip line. The correlation of the maximum dislocation density and hydrogen diffusion velocity is shown in Fig.10. The typical inverse pile-up of dislocations occurs when the velocities of hydrogen and dislocations are in competitive relationship $(0.03<\alpha<0.3)$, and it results in stress concentration due to high dislocation density. Therefore the dislocation pile-up type hydrogen embrittlement occurs. In contrast, when $\alpha$ takes a value other than this region, the maximum dislocation density rapidly decreases and the inverse pile-up of dislocations does not occur. This result indicates that the material with intermediate $\alpha(0.03<\alpha<0.3)$ causes the dislocation pile-up type hydrogen embrittlement with facet like transgranular fracture. Here the range of $\alpha$ corresponds to the condition in which hydrogen velocity is slower than that of the dislocation. Therefore the ductile material is supposed to satisfy this condition. Hence the occurrence of the facet like transgranular fracture under corrosive environment for ductile material is supposed to be dominated by the dislocation pile-up type hydrogen embrittlement. The hydrogen embrittlement mechanism mentioned here is different from the embrittlement mechanism for high strength steel with intergranular fracture, and the occurrence condition of this type embrittlement is dominated by the relative relationship between dislocation velocity and hydrogen diffusivity.

Based on these results, the occurrences of each fracture mechanism (anodic dissolution, hydrogen embrittlement with intergranular fracture and dislocation pile-up type transgranular facet like fracture) under corrosive conditions are dominated by the relative relationships between dislocation velocity and hydrogen diffusivity. Moreover, it is theoretically confirmed that anodic dissolution is dominant for ductile materials with lower yield stress, hydrogen accumulation is dominant for brittle materials with higher yield stress, and transgranular fracture caused by typical inverse pile-up of dislocations occurs for intermediate yield stress materials.

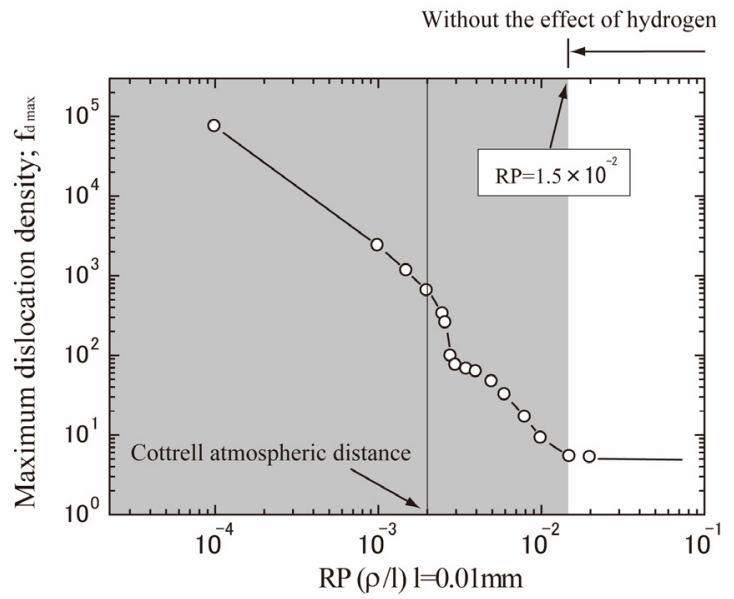

Fig.9 Correlation of the maximum dislocation density with the distance of hydrogen atom from slip line 


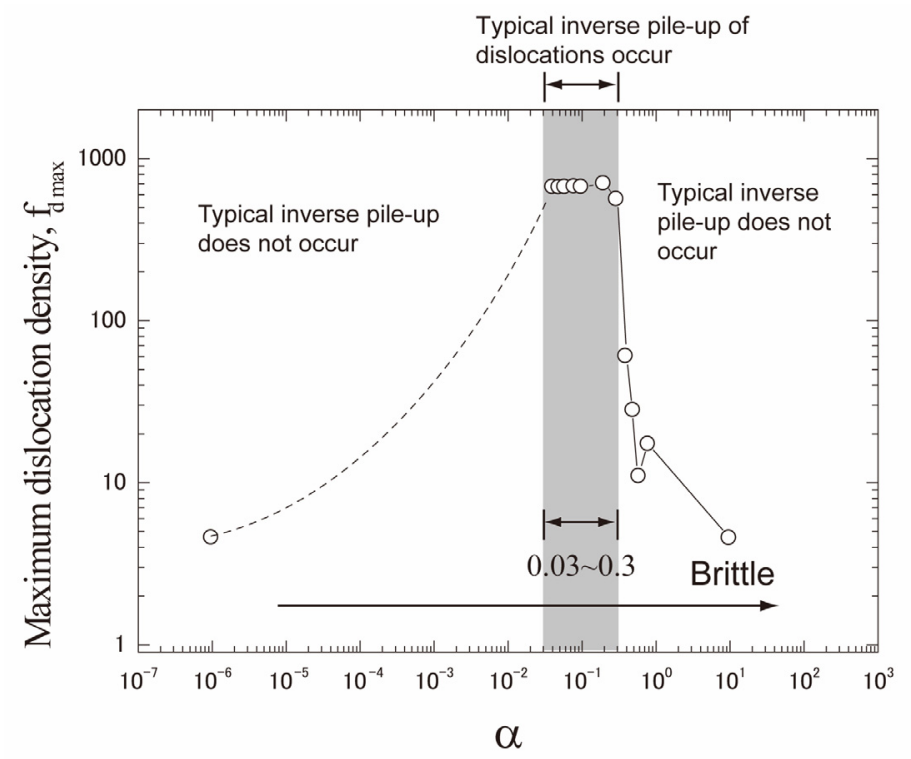

Fig.10 Correlation of the maximum dislocation density with the rate of hydrogen diffusion

\section{Conclusions}

The analysis of the mechanical interaction between dislocations emitted from a crack tip and the hydrogen atom around the crack tip was performed using the physical model of a crack with slip plane. The following conclusions were obtained.

1. Two-dimensional physical model around a mode I crack and dislocations located on the neighboring slip line is developed in order to investigate the mechanical interaction between them. In this physical model, the crack is replaced by infinitesimal continuum distribution of edge dislocations.

2. Analyses based on the physical model mentioned in conclusion 1 reveal the fracture mechanisms of anodic dissolution, intergranular fracture caused by hydrogen accumulation and transgranular fracture under corrosive condition are dominated by the relative relationship between the velocities of dislocations and hydrogen. Anodic dissolution is dominant for ductile materials with lower yield stress, hydrogen accumulation is dominant for brittle materials with higher yield stress, and transgranular fracture caused by typical inverse pile-up of dislocations occurs for intermediate yield stress materials. These results are in good agreement with the results of the interaction with mode II crack, dynamic dislocations and hydrogen based on one-dimensional analysis.

3. Based on the one-dimensional physical model (the validity is confirmed in conclusion 1 and 2 from the physical point of view), the occurrence conditions of the mechanical interaction between dislocations and hydrogen are estimated. When the distance between a hydrogen atom and the slip line is less than $1.5 \times 10^{-1} \mu \mathrm{m}$ and/or the velocity of hydrogen diffusion is $0.03 \sim 0.3$ times faster than the dislocation velocity, dislocations and a hydrogen atom are in the competitive relationship and have the mechanical interaction each other.

4. Conclusion 3 indicates the dislocation pile-up type hydrogen embrittlement occurs under the competitive mobility condition between them. It gives the theoretical evidence to the transgranular facet like fracture for ductile materials based on the interaction with dislocation and hydrogen. 


\section{Acknowledgement}

The partial support of this work by Grand-in-Aid for COE (Center of excellence) Research (No.11CE2003), The Ministry of Education, Culture, Sports, Science and Technology is gratefully acknowledged.

\section{References}

(1) Kohira, K., Yatake, T. and Yurioka, N., A Numerical Analysis of the Diffusion and Trapping of Hydrogen in Steels and its Application to Weldments, Journal of the Japan Welding Society, Vol.43, No.9, (1974), pp.921-930 (in Japanese).

(2) Satoh, K., Terasaki, T. and Yamashita, Y., Effect of Welding Conditions on Local Hydrogen Concentration at the Root of Weld Metal, Journal of the Japan Welding Society, Vol.48, No.7, (1979), pp.504-509 (in Japanese).

(3) Liu, H. W., Stress-Corrosion Cracking and Interaction between Crack-Tip Stress Field and solute Atoms, Journal of Basic Engineering, Vol.92, No.3, (1970), p.633.

(4) Van Leeuwen, H. P., Plateau Velocity of SCC in High Strength Steel -A Quantitative Treatment, Corrosion, Vol.31, No.2, (1975), pp.42-50.

(5) Sofronis, P. and Birnbaum, H. K., Mechanics of the Hydrogen-dislocation-Impurity Interactions .1. Increasing shear Modulus, Journal of the Mechanics and Physics of Solids, Vol.43, No.1, (1995), pp.49-90.

(6) Yokobori, A. T. Jr., Nemoto, T., Satoh, K. and Yamada, T., Numerical Analysis on hydrogen Diffusion and Concentration in Solid with Emission Around the Crack Tip, Engngineering Fracture Mechanics, Vol.55, No.1, (1996), pp.47-60.

(7) Yokobori, A. T. Jr., Chinda, Y., Nemoto, T., Satoh, K. and Yamada, T., The Characteristics of Hydrogen Diffusion and Concentration Around a Crack Tip Concerned with Hydrogen Embrittlement, Corrosion Science, Vol.44, (2002), pp.407-422.

(8) Yokobori, A. T. Jr., Uesugi, T., Sendoh, M. and Shibata, M., The Effect of Stress Wave Form on Corrosion Fatigue Crack Growth Rate on the Basis of Hydrogen Diffusion Theory, ,Strength Fracture and Complexity, An International Journal, Vol.1, (2003), pp.187-204.

(9) Magnin, T., Chieragatti, R and Oltra, B., Mechanism of Brittle-Fracture in a Ductile 316 Alloy During Stress-Corrosion, Acta Metallurgica et Materialia, Vol.38, No.7, (1990), pp.1313-1319.

(10) Yokobori, A. T. Jr., Isogai, T. and Yokobori, T.,A Model Emitting Dislocation Group From Crack-Tip with Stress Singularity and its Application to Brittle-Ductile Transition, Acta Metallurgica et Materialia, Vol.41, No.5, (1993), pp.1405-1411.

(11) Yokobori, A. T. Jr., Iwadate, T. and Isogai, T., Dislocation Emission and dynamics Under the Stress Singularity at the Crack Tip and Its Application to the Dynamic Loading Effect on Fracture Toughness, ASTM1207, (1994), pp.464-477.

(12) Yokobori, A. T. Jr., Taketomi, S., Ha, J. C. and Shoji, T. and Li, G. F., The Mechanism of Hydrogen Embrittlement on the Basis of the Interaction Model of Dislocations and Hydrogen around a Crack Tip, Strength, Fracture and Complexity, An Inernational Journal, Vol.2, (2004), pp.95-109.

(13) Taketomi, S., Yokobori, A. T. Jr. and Shoji, T., Mechanism of Hydrogen Embrittlement Due to the Interaction of a Crack, Moving Dislocations and Hydrogen Cluster, Key Engineering Materials, Vol. 261-263, (2004), pp.937-942.

(14) Yokobori, A. T. Jr., The Mechanism of Hydrogen Embrittlement : the Stress Interaction between a Crack, a Hydrogen Cluster, and Moving dislocations, International Journal of Fracture, Vol.128, (2004), pp.121-131. 
(15) Yokobori, T., Yoshida, M., Kuroda, H., Kamei, A and Konosu, S., Non-linear Interaction between Main Crack and Near-by Slip Band, Engingineering Fracture Mechanics, Vol.7, (1975), pp.377-388.

(16) Yokobori, T., Zairyo-Kyodogaku, (1974), Iwanami Press, p.55 (in Japanese).

(17) Taketomi, S and Yokobori, A. T. Jr., The Effect of the Interaction between Dislocations and Hydrogen Around a Crack Tip on Hydrogen Embrittlement Under Cyclic Loading Condition, Journal of Solid Mechanics and Materials Engineering, Vol.1, (2007), pp.126-139. 